\title{
SPL Miscellaneous Identifier Types Terminology
}

National Cancer Institute

\section{Source}

National Cancer Institute. SPL Miscellaneous Identifier Types Terminology. NCI

Thesaurus. Code C99288.

Terminology used for representation of miscellaneous data types in the framework of Structured Product Labeling documents. 